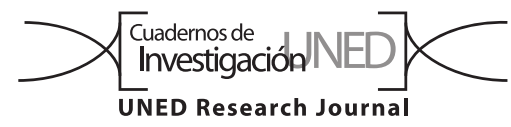

\title{
Modelo de vulnerabilidad ante incendios forestales para el Área de Conservación Guanacaste, Costa Rica
}

\author{
Daniela Vargas-Sanabria \& Carlos Campos-Vargas \\ Laboratorio de Investigación e Innovación Tecnológica, Vicerrectoría de Investigación, Universidad Estatal a Distancia, San José, 474-2050, \\ Costa Rica; dvargass@uned.ac.cr; ccamposv@uned.ac.cr
}

Recibido 09-III-2018 • Corregido 17-VII-2018 • Aceptado 19-VII-2018

\begin{abstract}
A wildfires vulnerability model for Guanacaste Conservation Area, Costa Rica. Wildfires occur in almost every type of ecosystem. However, vulnerability and propagation capacity of wildfires mostly relies on the amount of biomass and their water content. Particularly, Tropical dry forests are a vulnerable ecosystem to wildfires because, have at least six months with a precipitation of less than ten $\mathrm{mm}$ per month. Vulnerability assessment is a critical tool on wildfires management, defining the most vulnerable areas within the conservation area.The objective of this study was the implementation of a wildfire vulnerability model to identify priority areas for control and management of fire at Guanacaste Conservation Area (ACG). This model was designed integrating ecological components such as visual quality and biodiversity; and socio-economic components such as infrastructure and ecosystems services provided by ACG. Our results indicated that the most vulnerable areas at ACG contains a high density of species with pivotal ecological roles on ecosystem services. On the other hand, the less vulnerable areas are close to roads and communities. However, those last frequently experience wildfires. We expect that this model can be adapted in other protected wild areas with similar ecological and socio-economic characteristics to ACG and are often affected by wildfires taking into consideration the particularities present in each site.
\end{abstract}

Key words: vulnerability, wildfires, tropical dry forest, risk, geographic information systems, Guanacaste Conservation Area.
RESUMEN: Los incendios forestales ocurren en casi todos los tipos de ecosistemas; sin embargo, la vulnerabilidad y capacidad de programación de los incendios depende en gran medida de la cantidad de combustible y el contenido de humedad. Los bosques secos tropicales en particular son un ecosistema vulnerable a los incendios, ya que durante al menos 6 meses por año reciben una precipitación de menos a $10 \mathrm{~mm}$ por mes. La evaluación de la vulnerabilidad es una herramienta crítica en la prevención de incendios forestales, al estimar los sitios más vulnerables al fuego. El objetivo de este estudio fue implementar un modelo de vulnerabilidad ante incendios forestales que permita definir áreas prioritarias para el control del fuego en el Área de Conservación Guanacaste (ACG). El modelo se diseñó integrando componentes ecológicos como calidad visual y biodiversidad, y componentes socioeconómicos como infraestructura y servicios ecosistémicos presentes en el ACG. Nuestros resultados indican que las áreas más vulnerables a incendios forestales en el AGC existen una presencia alta de especies de alto valor ecológico y mayor desarrollo de servicios ecosistémicos. Por otro lado, las zonas que se encuentran cerca de comunidades y carreteras son menos vulnerables; sin embargo, presentan una mayor incidencia de incendios forestales. Se espera que este modelo puede ser adaptado en otras áreas silvestres protegidas con características ecológicas y socioeconómicas a las del AGC y que se vean afectadas por incendios forestales, tomando en consideración las particularidades de cada sitio.

Palabras clave: vulnerabilidad, incendios forestales, bosque seco tropical, riesgo, sistemas de información geográfica, Área de Conservación Guanacaste.
Los incendios forestales son un fenómeno global que surge en los registros geológicos poco tiempo después de la aparición de las plantas terrestres (Bowman et al., 2009). Ocurren en casi todo tipo de ecosistema en todo el planeta; sin embargo, la extensión y capacidad de propagación de los incendios forestales dependen en gran medida de la cantidad de combustible y de la humedad que contiene dicho combustible (Mondal \& Sukumar, 2016). En el trópico, los bosques secos tropicales y hábitats asociados, son ecosistemas altamente susceptibles a los incendios forestales debido a la existencia de una prolongada época seca con hasta 8 meses y con una precipitación menor a 100 mm (Calvo-Alvarado, SánchezAzofeifa \& Portillo-Quintero, 2013), a diferencia de ecosistemas más húmedos como los bosques lluviosos o páramos que son susceptibles al fuego, especialmente en condiciones de severa sequía (Meyn, White, Buhk \& Jentsch, 2007).

Los bosques secos representan aproximadamente, el $42 \%$ de los bosques tropicales (Van Bloem, Murphy 
\& Lugo, 2004). En el neotrópico estos ecosistemas existen como fragmentos del que alguna vez fue un único y continuo fragmento de bosque que se extendía desde México hasta el norte de Argentina (Portillo-Quintero \& Sanchez-Azofeifa, 2010). Condiciones climáticas benéficas y suelos con relativamente alta fertilidad y buenas propiedades edáficas han hecho de estos ecosistemas atractivos para el desarrollo de actividades como el establecimiento de asentamientos urbanos con altas densidades poblacionales, deforestación y tala selectiva, expansión agrícola y ganadería extensiva (CalvoAlvarado et al., 2013; Sánchez-Azofeifa, Calvo-Alvarado, Marcos do Espírito-Santo, Fernandes \& Powers, 2013), siendo el fuego el mayor elemento conductor en la degradación y conversión del uso de sus suelos (Otterstrom, Schwartz \& Velázquez-Rocha, 2006).

Durante los meses de la estación seca, la hojarasca y los desechos leñosos se acumulan a lo largo del suelo del bosque como resultado de los árboles caducifolios y los fuertes vientos alisios lo que crea condiciones ideales para la propagación de un incendio forestal (Otterstrom et al., 2006). Los rayos ocasionalmente provocan incendios en los bosques secos, pero son de baja intensidad, de pequeña escala y; por lo general, son rociados por las lluvias (Van Bloem et al., 2004). La mayoría de los incendios forestales en los bosques secos están vinculados a los incendios establecidos intencionalmente por los agricultores que están preparando los campos agrícolas o realizando el mantenimiento de los pastos y cazadores (Otterstrom et al., 2006).

El fuego influye en los patrones y procesos del ecosistema global, incluida la distribución y estructura de la vegetación, el ciclo del carbono y el clima (Bowman et al., 2009). Una vez que un bosque arde, aumenta la susceptibilidad al fuego debido al aumento del calentamiento solar a través del dosel fracturado, y los incendios recurrentes pueden volverse endémicos (Cochrane, 2001). Los incendios pueden alterar drásticamente la diversidad de especies, la estructura y la regeneración de estos bosques. Por ejemplo, las formaciones de bosque seco tropical a menudo se degradan a etapas de sucesión tempranas principalmente causando incendios (Schmerbeck, 2011). Específicamente, con cada incendio, los árboles de diámetro pequeño se dañan más fácilmente, incluso los árboles más grandes pueden verse afectados por incendios superficiales si los incendios son recurrentes, dado que los árboles en los bosques tropicales tienen corteza delgada y no están bien adaptados a los incendios (Elvidge et al., 2001). Además, a través del tiempo, las fuentes locales de semillas desaparecerán, las especies forestales maduras serán eliminadas localmente $y$ el grupo de colonizadores posteriores al incendio se reducirá a especies con dispersión de largo alcance (Cochrane et al., 1999).

La evaluación del riesgo es una parte crítica de la prevención de incendios forestales ya que los recursos de planificación previos al evento requieren de herramientas objetivas para controlar cuándo y dónde es más probable que ocurra un incendio o cuándo y dónde tendrá más efectos negativos (Chuvieco et al., 2014b). La evaluación de la vulnerabilidad ante incendios forestales debe considerar el daño causado por el fuego como una parte crítica de la evaluación del riesgo (Chuvieco et al., 2014a). El objetivo del presente estudio consiste en la implementación de un modelo de vulnerabilidad ante incendios forestales con variables ecológicas y socioeconómicas que permita identificar las zonas prioritarias para el control y manejo del fuego en el Área de Conservación Guanacaste (ACG).

\section{METODOLOGÍA}

Área de estudio: El área de estudio (Fig. 1) está ubicada en el Área de Conservación Guanacaste, Guanacaste Costa Rica. Específicamente en seis sectores del Área de Conservación Guanacaste, ubicada en el distrito Santa Elena, cantón La Cruz, provincia Guanacaste, Costa Rica. Estos sectores son: Santa Rosa, Murciélago, Santa Elena, Junquillal, El Hacha y Pocosol; y fueron seleccionados debido a su importancia ecológica, ya que albergan ecosistemas de bosque seco tropical que son afectados de manera recurrente por incendios forestales (Fig. 1). La temperatura media es de $25^{\circ} \mathrm{C}$ y recibe una precipitación media anual de $1500 \mathrm{~mm}$ que varía de 900 a 2600 $\mathrm{mm}$. La estación seca dura por un mínimo de 5-6 meses, por lo general se extiende desde fines de diciembre hasta mediados de mayo (Sánchez-Azofeifa et al., 2017; Li et al., 2017). Actualmente el paisaje del ACG es un mosaico de bosques secundarios, en diversas etapas de regeneración, con diferentes historias de uso de la tierra (Li et al., 2018; Hilje et al., 2015). Históricamente, la introducción de la hierba Jaragua (Hyparrhenia rufa) del este de África alrededor de 1940 para crear pastizales para el ganado aumentó un intenso proceso de deforestación, tradicionalmente asociado con incendios antropogénicos (Janzen, 2000).

Componentes de vulnerabilidad: Para la generación del modelo de vulnerabilidad se tomó como base el diagrama de riesgo que contempla los factores de peligro y de vulnerabilidad diseñado por Chuvieco et al. (2014b) y adaptado para el sitio de estudio dentro del ACG, 

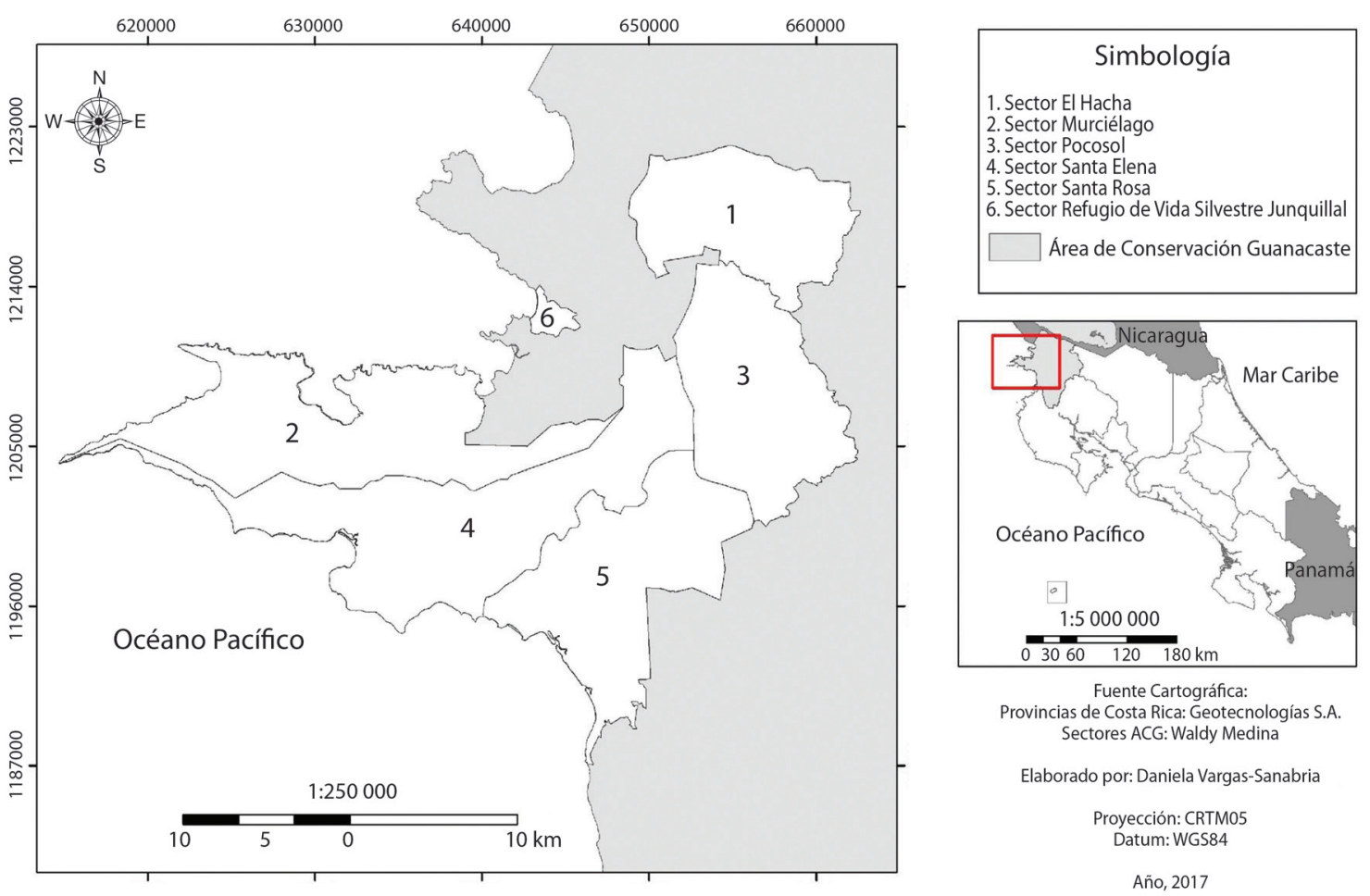

Fig. 1. Ubicación del área de estudio.

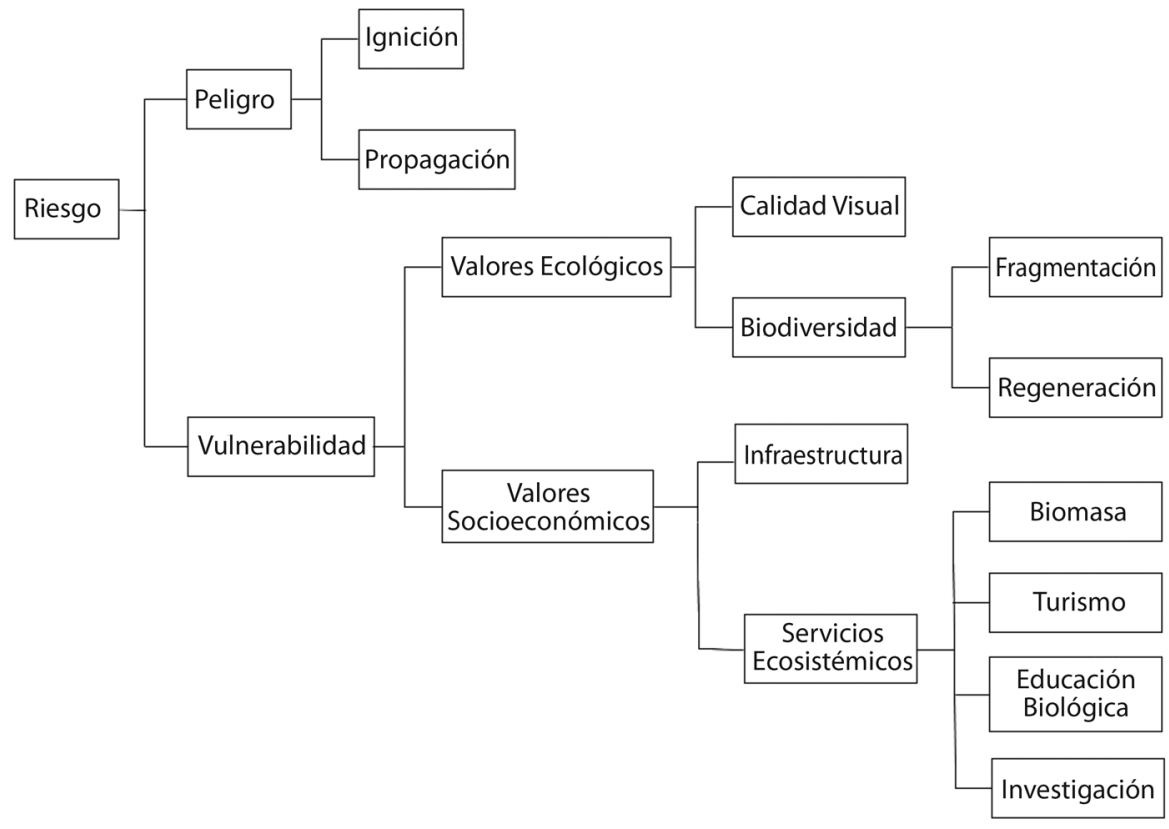

Fig. 2. Diagrama de riesgo adaptado de Chuvieco et al. (2014b) (Vargas-Sanabria, 2016). 
evaluándose solamente la vulnerabilidad, en específico los componentes ecológicos y socioeconómicos (Fig. 2).

El modelo se desarrolló en tres etapas: la primera consistió en la elaboración de la base de datos para cada variable de los componentes, estos datos fueron categorizados en un rango asociado a vulnerabilidad alta, media y baja. La segunda etapa consistió en la asignación de pesos según una evaluación multi-criterio basada en una encuesta que se realizó a cinco personas entre ellas personal del área de conservación e investigadores con criterio experto sobre la situación del fuego en el ACG, cada persona asignó a cada variable un valor de 1 a 10 (siendo 1 el valor más bajo y 10 el más alto), dependiendo de la importancia de la variable dentro del área protegida; y finalmente la etapa tres consistió en la integración de las variables para obtener como producto un modelo geoespacial con áreas asociadas a vulnerabilidad alta, media y baja. Los resultados obtenidos en cada etapa de la elaboración del modelo fueron validados por el personal del ACG.

\section{Valores ecológicos:}

\section{Calidad visual}

Se identificaron y georeferenciaron con equipo de posicionamiento global (Garmin 64 GPSmap, con una precisión de 3 metros) 25 puntos de interés establecidos por el personal del ACG como áreas prioritarias por su calidad visual (Fig. 3). Cada punto está asociado a dos parámetros: altura del observador (se estimó en 2 metros) y radio (se estimó en $20 \mathrm{~km}$ a la redonda, distancia que el ojo humano puede enfocar hasta el horizonte). Para analizar estos datos se utilizó la herramienta Viewshed del 3D Analyst Tools del programa ArcGis 10.3. Los valores dados por la herramienta fueron de 0 a 7; donde 0 representa áreas no visibles que responde a una vulnerabilidad baja, el valor de 1 a 3,5 se asignó como áreas con vulnerabilidad alta y de 3,6 a 7 áreas con vulnerabilidad media.

\section{Biodiversidad}

Dentro de la biodiversidad del paisaje se consideraron dos variables: fragmentación de hábitat y regeneración de estadios sucesionales. Para determinar el nivel de sucesión de las especies se establecieron tipos de vegetación dentro del área de estudio, tomando como base los datos obtenidos por Arroyo-Mora et al. (2005) e Hilje et al. (2015) y de acuerdo al mapa de cobertura de la tierra elaborado mediante la implementación de un sistema multi-algoritmo por Vargas-Sanabria \& Campos-Vargas (2018). Para asignar los años de regeneración por estadio se tomó como referencia y se adaptó el modelo hipotético de la secuencia ocurrida durante el crecimiento del bosque secundario de Guariguata y Ostertag (2001) (Cuadro 1).

En el modelo de vulnerabilidad se establece que las áreas con baja fragmentación son más vulnerables. Se

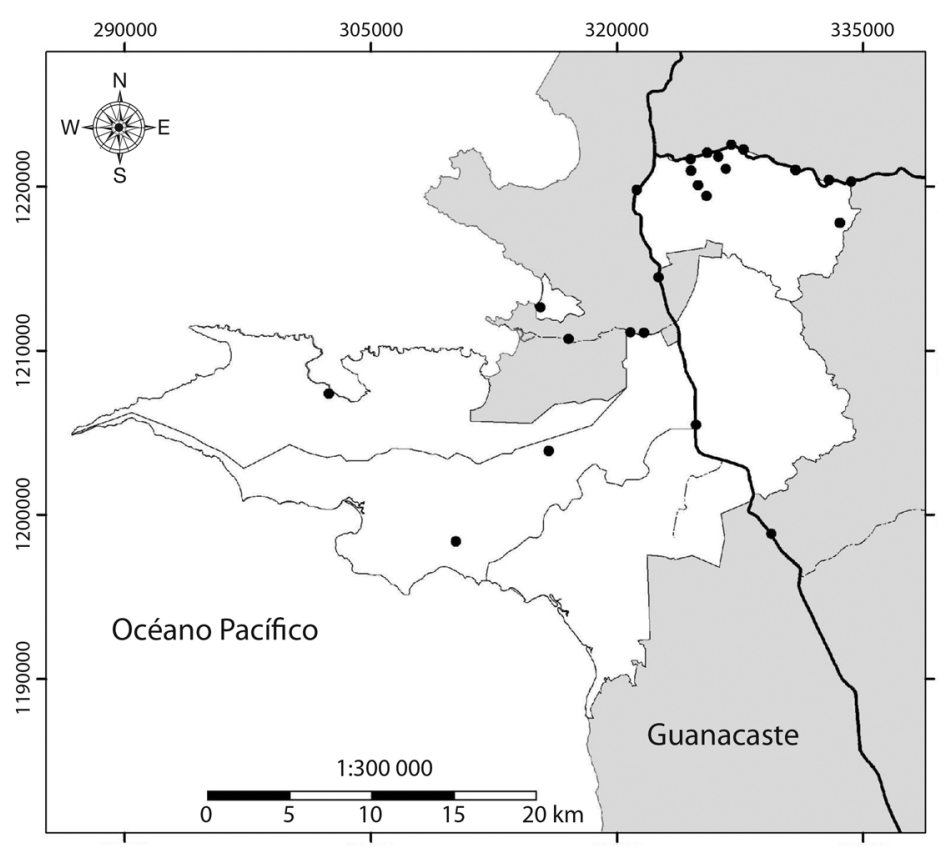

Fig. 3. Ubicación de puntos de interés. 
CUADRO 1

Secuencia temporal de la sucesión de hábitats adaptado de Guariguata \& Ostertag (2001).

\begin{tabular}{lcc}
\multicolumn{1}{c}{ Cobertura } & Tiempo en sucesión & Rango de vulnerabilidad \\
Sabanas y pastos & $1-5$ años & Bajo \\
$\begin{array}{l}\text { Bosque tempranos } \\
\text { Bosque secundarios, bosque de galería, manglar, bosques tardíos }\end{array}$ & $5-20$ años & Medio \\
y bosque seco enano & $20-100$ años & Alto \\
\hline
\end{tabular}

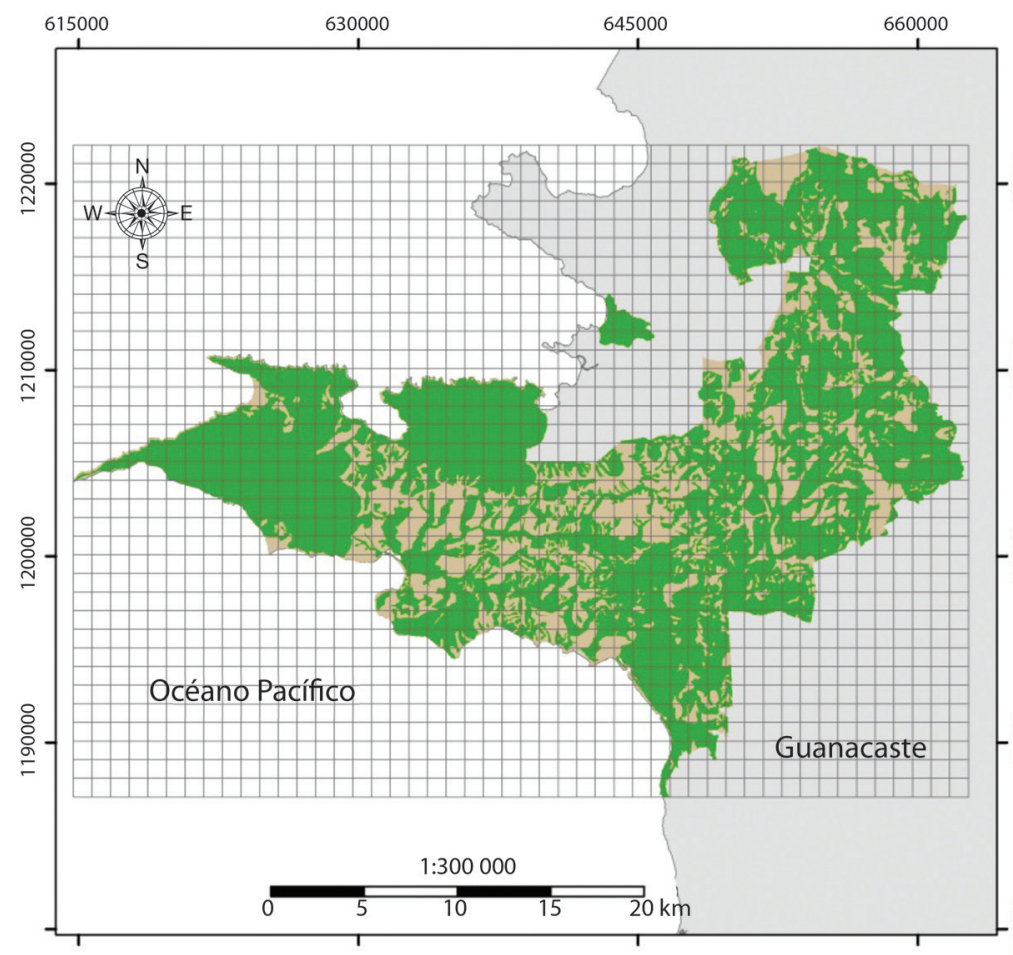

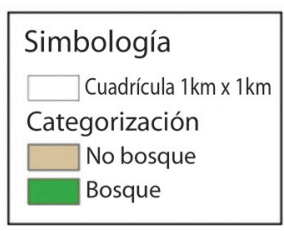

Diagrama de Ubicación

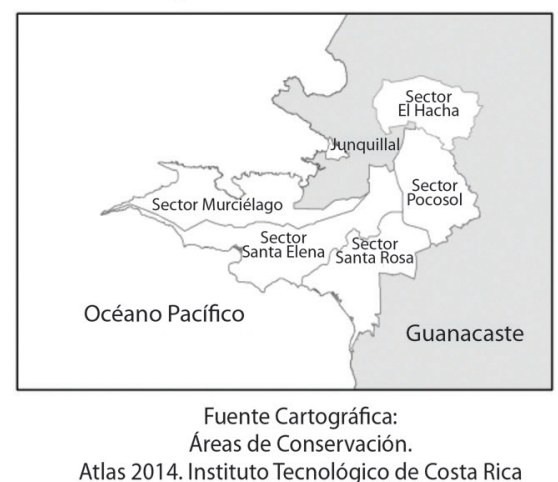

Elaborado por: Daniela Vargas-Sanabria

$$
\begin{aligned}
& \text { Proyección: CRTM05 } \\
& \text { Datum: WGS84 }
\end{aligned}
$$

Año, 2016

Fig. 4. Mapa de distribución de hábitats fragmentados.

procedió a caracterizar la cobertura de la tierra en dos clases bosque y no bosque; y se diseñó una cuadrícula con la herramienta Create Fishnet del programa ArcGis 10.3, con las dimensiones de $1 \mathrm{~km} \times 1 \mathrm{~km}$ (este valor fue validado por el personal del ACG), para localizar los parches más homogéneos y heterogéneos de cobertura (Fig. 4).

Posteriormente se realizó el análisis de fragmentación mediante la herramienta Zonal Statistics del programa ArcGis 10.3, calculando las estadísticas para cada zona (cuadrícula $1 \mathrm{~km} \times 1 \mathrm{~km}$ ) de acuerdo a la información de los valores (clases de bosque y no bosque). La clasificación de la vulnerabilidad de acuerdo a los valores de fragmentación se adaptó según lo establecido por el índice de fragmentación de Díaz (2003) (Cuadro 2).
CUADRO 2

Rango de vulnerabilidad en áreas fragmentadas adaptado de Díaz (2003).

\begin{tabular}{cc} 
Fragmentación & Rango de vulnerabilidad \\
$1>F \geq 0,7$ & Alto \\
$0,7>F \geq 0,5$ & Medio \\
$0,5>F$ & Bajo \\
\hline
\end{tabular}

\section{Valores socioeconómicos:}

\section{Infraestructura}

Se realizó un levantamiento cartográfico de las edificaciones propiedad del Sistema Nacional de Áreas de Conservación (SINAC) dentro del área de estudio 


\section{CUADRO 3}

Biomasa aérea en categorías de cobertura de la tierra para los sectores de estudio del ACG.

\begin{tabular}{lcc}
\multicolumn{1}{c}{ Cobertura } & $\begin{array}{l}\text { Biomasa } \\
\text { (Ton*ha) }\end{array}$ & \multicolumn{1}{c}{ Referencia } \\
$\begin{array}{l}\text { Bosque natural (bosque } \\
\text { de galería, bosque enano }\end{array}$ & 248,5 & Imbach et al. (2005) \\
$\begin{array}{l}\text { seco, bosque tardío) } \\
\text { Manglar }\end{array}$ & 229,5 & Imbach et al. (2005) \\
Bosque secundarios & 99,1 & Imbach et al. (2005) \\
Bosque temprano & 13,1 & Ulate, (2011) \\
Pasturas & 2,9 & Imbach et al. (2005) \\
\hline
\end{tabular}

mediante una imagen satelital Landsat del año 2014 del servicio web brindado por Google Earth. Para este caso la clasificación de los datos tomó en consideración solamente dos rangos de vulnerabilidad, alta y media, esto de acuerdo al criterio experto del personal del ACG.

\section{Servicios ecosistémicos}

Para determinar la biomasa aérea, se tomó como base el mapa de cobertura de la tierra elaborado por Vargas-Sanabria y Campos-Vargas (2018), referencias bibliográficas y consulta al personal del ACG. Los valores de biomasa para las categorías de cobertura de la tierra fueron descritos por Imbach, Coto y Salinas (2005) y Ulate (2011) (Cuadro 3).

Los componentes Turismo, Investigación y Educación biológica se analizaron según los sectores administrativos definidos por el ACG. En específico el componente Turismo se estimó tomando en cuenta el informe de visitación turística del parque a partir del año 2013 (Martínez \& Rodríguez, 2014). Por otro lado, Investigación tomó como base el número de proyectos de investigación por sector del 2004 al 2012. Por último, Educación biológica tomó como base los datos del Programa de Educación Biológica y la cantidad de estudiantes participantes en cada sector para el año 2015. En el Cuadro 4 se presentan los rangos de vulnerabilidad asociados a los valores seleccionados para cada variable dentro del componente de servicios ecosistémicos.

Asignación de pesos: Para identificar la importancia de las variables dentro del área silvestre protegida se asignó un valor a cada una a través de una evaluación multi-criterio realizada al personal del ACG e investigadores, de acuerdo a lo establecido por Vargas-Sanabria (2016). Posteriormente se asignó a cada variable el valor porcentual obtenido de la evaluación multi-criterio utilizando la Ecuación 1:

$V=\left[\left(F^{*} 0,19\right)+\left(B^{*} 0,12\right)+\left(C V^{*} 0,10\right)+\left(I^{*} 0,10\right)+\left(T^{*} 0,10\right)+\left(I n v^{*} 0,10\right)+\left(R^{*} 0,22\right)+\left(E B^{*} 0,08\right)\right]$

Dónde: V=Vulnerabilidad; F = Fragmentación; $\mathrm{B}=$ Biomasa; $\mathrm{CV}=$ Calidad visual; I = Infraestructura; $\mathrm{T}=$ Turismo; Inv= Investigación; $\mathrm{R}=$ Regeneración; $\mathrm{EB}=$ Educación biológica.

\section{CUADRO 4}

Rango de vulnerabilidad de acuerdo a las variables del componente de valores socioeconómicos.

\begin{tabular}{llc}
\multicolumn{1}{c}{ Variable } & \multicolumn{1}{c}{ Valores } & Rango de vulnerabilidad \\
Infraestructura & Casona & Alta \\
Biomasa & Edificaciones del SINAC y áreas circundantes & Baja \\
& $>100$ ton/ha & Alta \\
& $>40-100$ ton/ha & Medio \\
Turismo & $0-40$ ton/ha & Bajo \\
& $>10000$ visitas & Alta \\
Investigación & $>5000-10000$ visitas & Media \\
& $0-5000$ visitas & Baja \\
& $>21$ investigaciones & Alta \\
Educación Biológica & $11-20$ investigaciones & Media \\
& $0-10$ investigaciones & Baja \\
& $150-300$ estudiantes & Alta \\
& $0-150$ estudiantes & Media \\
\hline
\end{tabular}


Integración de componentes de vulnerabilidad: Todas las variables fueron sumadas por medio de la herramienta calculadora raster del programa ArcGis 10.3, mediante el siguiente proceso: primero se sumaron las variables de servicios ecosistémicos e infraestructura y se obtuvo los valores socioeconómicos. Posterior a esto se sumó las variables de regeneración y fragmentación que generaron el componente de biodiversidad y este se sumó con la calidad visual y se obtuvieron los valores ecológicos. Por último, se sumaron los valores ecológicos y socioeconómicos; y se generó el modelo final de vulnerabilidad.
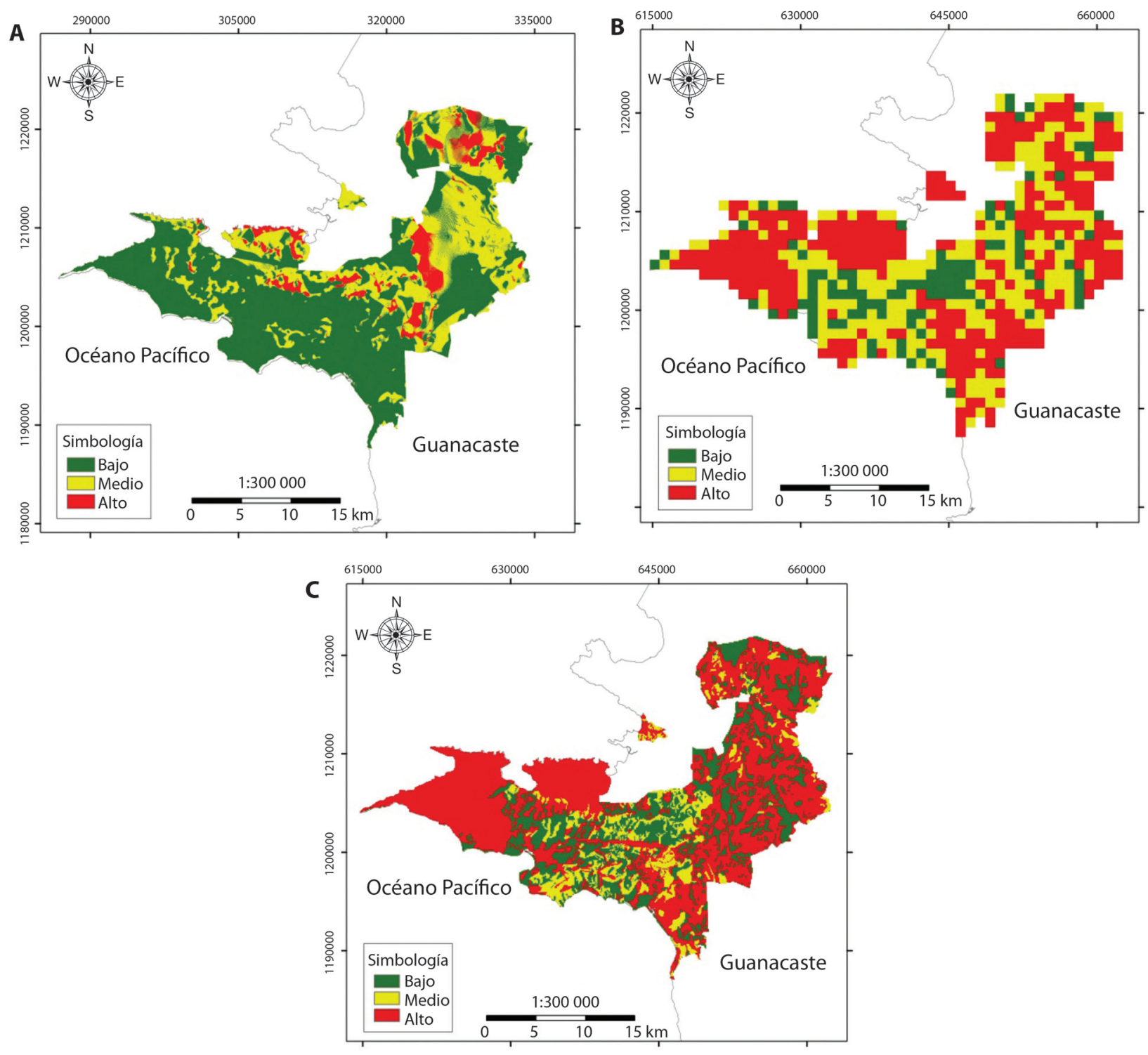

Fig. 5. A) Nivel de vulnerabilidad en la calidad del paisaje. B) Nivel de vulnerabilidad en áreas fragmentadas. C) Nivel de vulnerabilidad en la regeneración de especies ante incendios forestales (Vargas-Sanabria, 2016).

\section{Valores ecológicos: Los valores obtenidos en la ca- lidad visual muestran rangos de vulnerabilidad alta en puntos donde la visibilidad está asociada con áreas de interés para el área protegida, mientras que las áreas con rangos de vulnerabilidad media y baja tienen poca atracción visual por la topografía y por la restricción de uso solo para el personal del ACG (Fig. 5A). Las áreas con menor fragmentación y más homogenizadas son las más vulnerables a incendios forestales, mientras que los ran- gos de vulnerabilidad media o baja representan áreas \\ RESULTADOS}

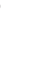

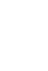

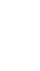


más heterogéneas, que por lo general se componen de parches de cobertura vegetal como sabanas, pastos, bosques tempranos y bosques intermedios, además son áreas con alta incidencia de incendios forestales (Fig. 5B).

En la Figura $5 \mathrm{C}$ se representa la vulnerabilidad de acuerdo al análisis del tiempo en la regeneración de las especies. Se determinó que los estadios sucesionales avanzados como bosques de galería, bosques intermedios y bosques tardíos tienen una alta vulnerabilidad; mientras que las áreas con vulnerabilidad media y baja presentan estadios sucesionales menos avanzados como bosques tempranos, sabanas y pastos.

Valores Socioeconómicos: Se obtuvo valores de vulnerabilidad alta en el área donde se encuentra la edificación de la Casona Santa Rosa (Sitio Patrimonio de la Humanidad), mientras que otros tipos de infraestructuras dentro del área y zonas circundantes propiedad del SINAC, presentan valores de vulnerabilidad media (Fig. 6). Dentro de los servicios ecosistémicos, la distribución de biomasa aérea se localiza en mayor proporción en los sectores más impactados por incendios forestales con rangos de vulnerabilidad alta; mientras que las áreas con biomasa asociada a coberturas de bosques intermedios presentan una vulnerabilidad media y las áreas con bajo contenido de biomasa una vulnerabilidad baja (Fig. 7A).

El turismo representa un importante insumo económico en el área protegida, es por ello que las áreas con más afluencia turística representan una vulnerabilidad alta, mientras que las áreas con poco desarrollo turístico tienen media y baja vulnerabilidad (Fig. 7B). Dentro del sector Santa Rosa se localiza el Centro de Estudios del Bosque Tropical Seco, lo que provoca una alta afluencia de investigadores y al ser uno de los sectores con menos incendios reportados, es por ello que representa un sector con alta vulnerabilidad. En los sectores con media y baja vulnerabilidad se desarrolla poco la investigación y se da más presencia de incendios forestales (Fig. 7C). Por otro lado, los proyectos de educación biológica han representado un importante insumo en la prevención de incendios forestales. Al igual que en las actividades de turismo e investigación el sector Santa Rosa se ve constantemente visitado por estudiantes del programa, es por esto que representa un área con alta vulnerabilidad, mientras que los sectores con media y baja vulnerabilidad reciben menos estudiantes y desarrollan menos programas educativos (Fig. 7D).
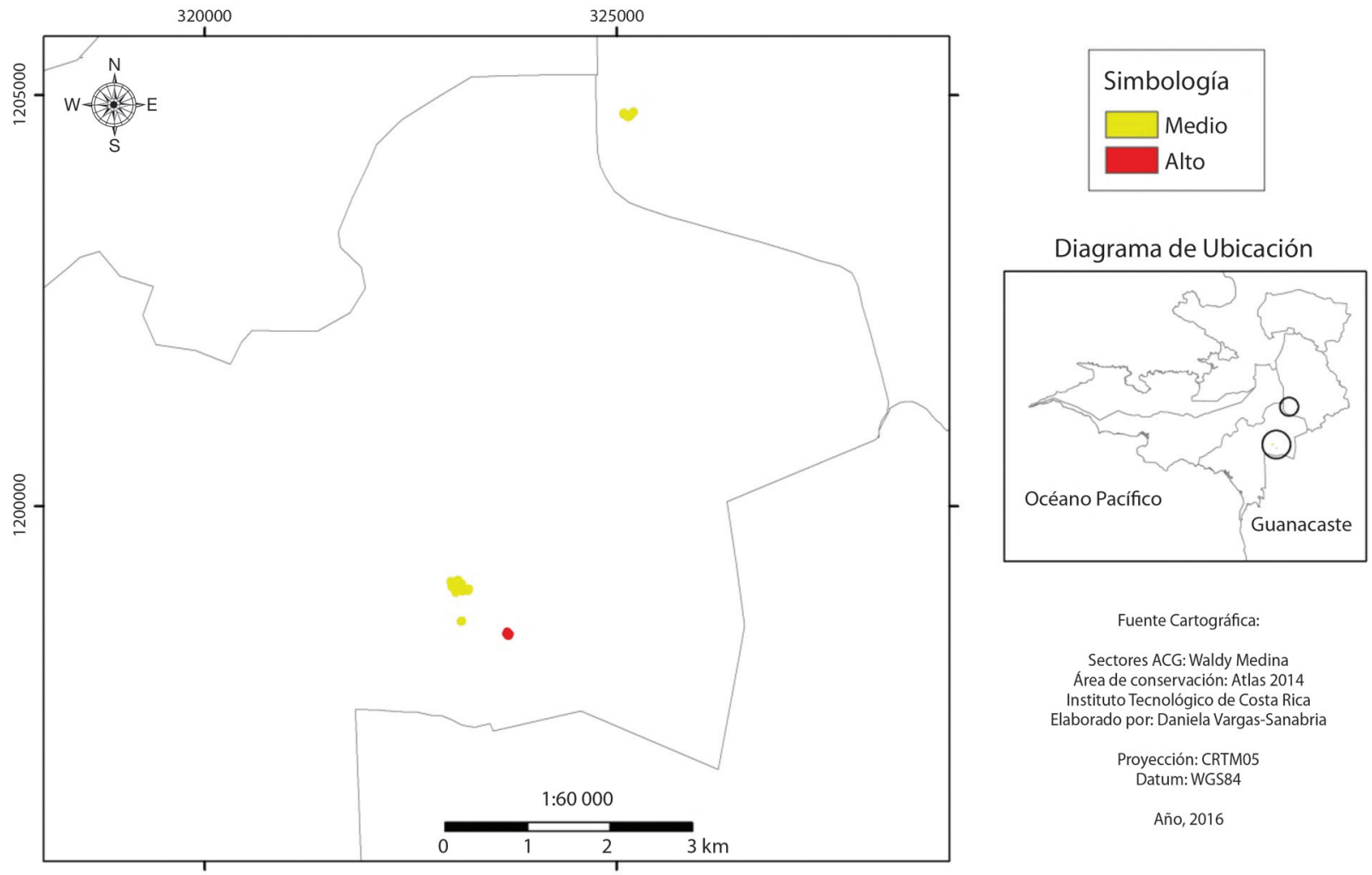

Fig. 6. Nivel de vulnerabilidad en la infraestructura ante Incendios Forestales en el Área de Conservación Guanacaste, Costa Rica, 2016. 

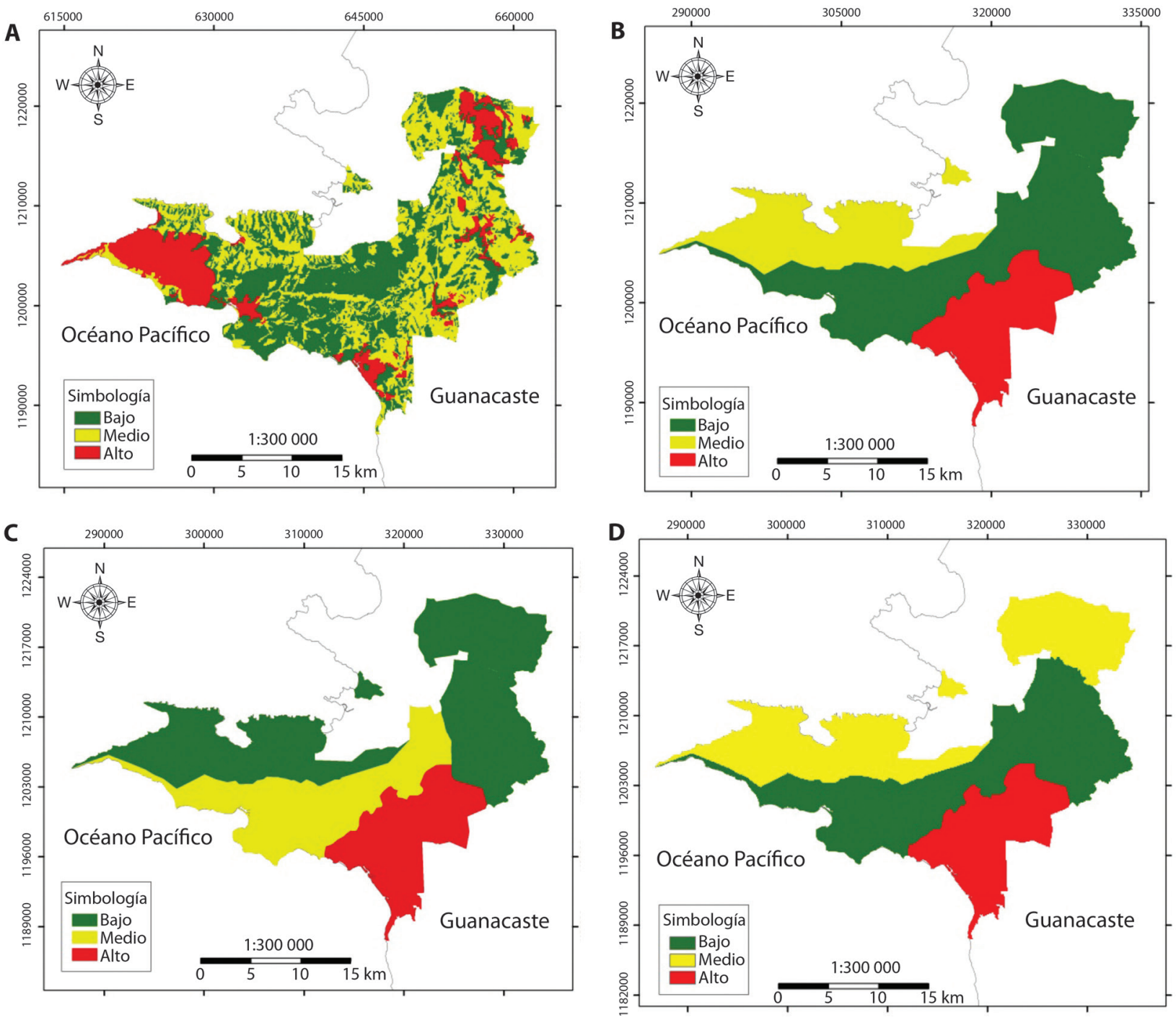

Fig. 7. A) Nivel de vulnerabilidad en biomasa aérea. B) Nivel de vulnerabilidad en visitación turística. C) Nivel de vulnerabilidad en investigación. D) Nivel de vulnerabilidad en programas de educación biológica (Vargas-Sanabria, 2016).

\section{Integración de variables}

En la Figura 8 se muestra el modelo de vulnerabilidad resultante de la integración de todas las variables mencionadas anteriormente. Las áreas con mayor vulnerabilidad corresponden a las regiones donde se han dado pocos o ningún incendio forestal, mientras que las áreas que han sido afectadas de forma recurrente por incendios forestales son menos vulnerables. En estas áreas hay poca biodiversidad y por lo general los ecosistemas están adaptados al fuego y regeneran de forma más rápida. Se detectó que en estas áreas hay poca influencia de actividades socioeconómicas que puedan afectar el área protegida tras el evento de un incendio forestal.

\section{DISCUSIÓN}

La evaluación de la vulnerabilidad ante incendios forestales es muy importante para el análisis de los efectos del fuego sobre los valores ecológicos y socioeconómicos. Este tipo de modelos proporcionan una herramienta de alto valor en la estimación de daños y pérdidas en áreas que son afectadas de forma recurrente por incendios forestales (Chuvieco et al., 2014a). Por otro lado, el paisaje del ACG refleja una variabilidad de sucesiones vegetales controladas en su mayoría por la historia de uso de la tierra en el área, la influencia de la vegetación remanente posterior al abandono del lugar (Leiva, Rocha, 


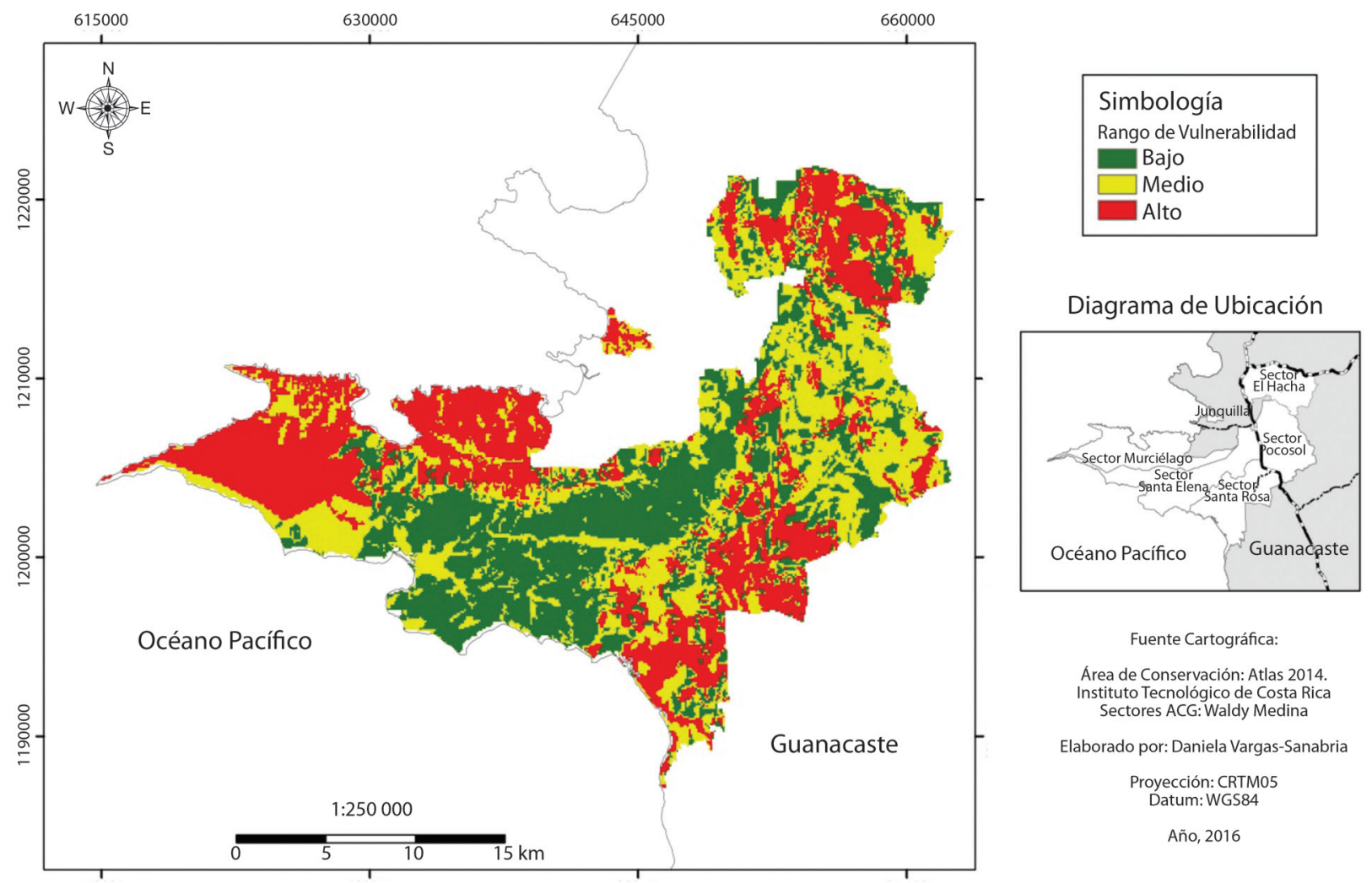

Fig. 8. Zonas vulnerables ante incendios forestales en el Área de Conservación Guanacaste, Costa Rica, 2016.

Mata \& Gutiérrez-Soto, 2009) y la dinámica de los incendios forestales.

Las áreas con vulnerabilidad alta se encuentran dominadas por especies de regeneración lenta y hábitats con baja fragmentación, lo que indica la presencia de especies de alto valor ecológico las cuales han sido capaces de adaptarse a condiciones climáticas, topográficas y edáficas, donde la presencia de incendios forestales es mínima. No obstante, en los ecosistemas donde hay presencia de coberturas de sabanas y pastos, conformados generalmente por Bulbostylist paradoxa -especie adaptada para sobrevivir al fuego y responder de forma productiva (Myers, 2006)-, la regeneración se presenta pocos meses después de un incendio forestal. La recurrencia de incendios forestales en ecosistemas causa considerables niveles de fragmentación de nuevos hábitats, esto propicia la generación de nuevos incendios, lo que ocasiona una alteración en los regímenes normales del fuego (Fernández et al., 2010). La recurrencia de estos eventos reduce de manera significativa el tamaño y la densidad de fragmentos que corresponden a bosques no quemados, destruyendo la vegetación que se encuentra en regeneración y propiciando la colonización de especies como las hierbas o pastos, las cuales aumentan la probabilidad de incendios forestales. Esto también se asocia con la muerte de individuos arbóreos y la acumulación de materia seca en los bordes de los fragmentos generando material combustible (Cochrane, 2003).

La mayor cantidad del material combustible se acumula cerca de carreteras y poblados propiciando el inicio de un incendio, aunque en Costa Rica los incendios forestales no han alcanzado aún la interfaz urbana como en España, Portugal o Grecia, donde la afectación directa a viviendas es constante por incendios forestales; $y$ las pérdidas humanas y monetarias ascienden de forma considerable (Birot, 2009). Por lo general, las estadísticas relacionadas a las consecuencias de un incendio forestal solo toman en cuenta los valores de bienes de mercado, sin considerar los servicios ecosistémicos, tales como biodiversidad, biomasa, valor histórico y cultural del área protegida (Birot, 2009). Por ejemplo, la reducción del ecosistema en términos de pérdidas potenciales en la reducción de sumideros de carbono y biomasa que se encuentran almacenados en el bosque, las pérdidas de oportunidades de ocio en medio del ambiente y las pérdidas de bienes no maderables son menos relevantes en términos de valores socioeconómicos, 
pero tienen un importante efecto en la economía local (Chuvieco et al., 2014b).

Sin embargo, en los sectores donde la vulnerabilidad es alta, los valores socioeconómicos presentan constante actividad por servicios ecosistémicos e infraestructuras. En esta investigación no se estimó la pérdida económica de estos bienes y servicios por falta de información, pero se estima que un eventual incendio forestal dentro del sector Santa Rosa generaría pérdidas económicas muy importantes para el área protegida, a nivel local, regional y nacional; esto debido a que el sector Santa Rosa presenta la mayor afluencia de turistas, desarrollo de investigaciones, educación biológica y edificaciones con importante historia para el país.

En definitiva, los modelos de vulnerabilidad a pequeña escala proporcionan mucha información para complementar modelos más complejos que se han realizado a nivel mundial como el diseñado por Chuvieco et al., (2014a); y en donde la recopilación de datos fiables ha sido imposible. Esta es una de las razones por lo cual, la validación de los modelos con criterio experto es un gran aporte en la integración de bases de datos, generando modelos con mayor detalle y adaptados a un escenario mucho más real de la situación de vulnerabilidad a incendios forestales que enfrentan las áreas protegidas, en especial el Área de Conservación Guanacaste.

\section{AGRADECIMIENTOS}

Los autores externan su agradecimiento a la Vicerrectoría de Investigación de la Universidad Estatal a Distancia (UNED) y al Acuerdo de Mejoramiento Institucional (AMI) por su apoyo financiero para la realización de las giras y actividades de capacitación. A la Comisión de Movilidad Internacional Estudiantil por la beca otorgada para la pasantía académica a España y al Programa de Investigación para la Promoción del Trabajo en Red (PRORED) por el apoyo económico para realizar giras de campo. Al Centro Nacional de Alta Tecnología y al Laboratorio PRIAS por el espacio y apoyo económico brindado para la realización del proyecto. Así mismo, al Área de Conservación Guanacaste y al personal del Programa de Control y Protección de Incendios Forestales, en especial a Didi Guadamuz y Julio Díaz. Además, se extiende un especial agradecimiento al Dr. Emilio Chuvieco Salinero y a todos los investigadores del Departamento de Geografía y Grupo de Teledetección Ambiental de la Universidad Alcalá de Henares, España por toda su colaboración y capacitación.

\section{REFERENCIAS}

Arroyo-Mora, J. P., Sánchez-Azofeifa, G. A., Kalacska, M. E. R., Rivard, B., Calvo-Alvarado, J., \& Janzen, D. H. (2005). Secondary Forest Detection in a Neotropical Dry Forest Landscape Using Landsat 7 ETM + and IKONOS Imagery. Biotropica, 37 (4), 497-507. doi:10.1111/j. 1744-7429.2005.00068

Birot, Y. (Ed.). (2009). Convivir con los incendios forestales: lo que nos revela la ciencia. Torikatu: European Forest Institute.

Bowman, D. M. J. S., Balch, J. K., Artaxo, P., Bond, W. J., Carlson, J. M., Cochrane, M. A., \& Pyne, S. J. (2009). Fire in the earth system. Science, 324(5926), 481-484. doi:10.1126/ science. 1163886

Calvo-Alvarado, J., Sánchez-Azofeifa, A., \& Portillo-Quintero, C. (2013). Neotropical Seasonally Dry Forests. Encyclopedia of Biodiversity, 5, 488-500. doi: 10.1016/ B978-0-12-384719-5.00354-3

Chuvieco, E., Martinez, S., Roman, M. V., Hantson, S., \& Pettinari, M. L. (2014a). Integration of ecological and socio-economic factors to assess global vulnerability to wildfire. Global Ecology and Biogeography, 23(2), 245-258. doi:10.1111/geb.12095

Chuvieco, E., Aguado, I., Jurdao, S., Pettinari, M. L., Yebra, M., Salas, J., Hantson, S., de la Riva, J., Ibarra, P., Rodrigues, M., Echeverría, M., Azqueta, D., Román, M. V., Bastarrika, A., Martínez, S., Recondo, C., Zapico, E., \& Martínez-Vega, F. J. (2014b). Integrating geospatial information into fire risk assessment. International Journal of Wildland Fires, 23, 606-619. doi: 10.1071/WF12052

Cochrane, M. A., Alencar, A., Schulze, M. D., Souza, C. M., Nepstad, D. C., Lefebvre, P., \& Davidson, E. A. (1999). Positive feedbacks in the fire dynamic of closed canopy tropical forests. Science, 284(5421), 1832-1835. doi:10.1126/ science.284.5421.1832

Cochrane, M. A. (2001). Fire in Evergren and Tropical. Conservation Biology, 15(6), 1515-1521.

Cochrane, M. A. (2003). Fire Science for rainforest. Nature, 421, 913-919.

Díaz, A. (2003). Instrumentos para la planificación integral del uso de la tierra con sistemas de información geográficaun caso de estudio en Argentina. Recuperado de http:// edoc.hu-berlin.de/dissertationen/diaz-lacava-amalia-nahir-2003-07-16/PDF/Diaz-Lacava.pdf.

Elvidge, C. D., Hobson, V. R., Baugh, K. E., Dietz, J. B., Shimabukuro, Y. E., Krug, T., \& Echavarria, F. R. (2001). DMSP-OLS estimation of tropical forest area impacted by surface fires in Roraima, Brazil: 1995 versus 1998. International Journal of Remote Sensing, 22(14), 26612673. doi:10.1080/01431160120888

Fernández, I., Morales, N., Olivares, L., Salvatierra, J., Gómez, M., \& Montenegro, G. (2010). Restauración ecológica para ecosistemas nativos afectados por incendios forestales. Pontificia Universidad Católica de Chile. Santiago. Chile. 
Guariguata, M. R., \& Ostertag, R. (2001). Neotropica secondary forest succesion: changes in structural and functional characteristics. Forest Ecology and Management, 148, 185-206.

Imbach, P., Coto, O., \& Salinas, Z. (2005). Valoración de los residuos biomásicos en Costa Rica usando Sistemas de Información Geográfica. CATIE. Turrialba, Costa Rica.

Janzen, D. H. (2000). Costa Rica's Area de Conservación Guanacaste: A long march to survival through non-damaging biodevelopment. Biodiversity, 1(2), 7-20. doi:10. 1080/14888386.2000.9712501.

Hilje, B., Calvo-Alvarado, J., Jiménez-Rodríguez, C., \& SánchezAzofeifa, A. (2015). Tree Species Composition, Breeding Systems, and Pollination and Dispersal Syndromes in Three Forest Successional Stages in a Tropical Dry Forest in Mesoamerica. Tropical Conservation Science, 8(1), 7694. doi:10.1177/194008291500800109

Leiva, J., Mata, R., Rocha, O., \& Gutiérrez, M. (2009). Cronología de la regeneración del bosque tropical seco en Santa Rosa, Guanacaste, Costa Rica. I. Características Edáficas. Revista de biología tropical, 57(3), 801-815.

Li, W., Campos-Vargas, C., Marzahn, P., \& Sanchez-Azofeifa, A. (2018). "On the Estimation of Tree Mortality and Liana Infestation Using a Deep Self-Encoding Network." International Journal of Applied Earth Observation and Geoinformation, 73(May), 1-13. doi:10.1016/j. jag.2018.05.025

Li, W., Campos-Vargas, C., Marzahn, P., \& Sanchez-Azofeifa, A. (2017). "Identifying Tropical Dry Forests Extent and Succession via the Use of Machine Learning Techniques." International Journal of Applied Earth Observation and Geoinformation, 63(July),196-205. doi:10.1016/j. jag.2017.08.003

Martínez, R., \& Rodríguez, D. (Abril de 2008). Los incendios forestales en México y América Central. Memorias del Segundo Simposio Internacional Sobre Políticas, Planificación y Economía de los Programas de Protección Contra Incendios Forestales: Una Visión Global. Córdoba, España. Recuperado de http://www. fs.fed.us/psw/publications/documents/psw_gtr208es/ psw_gtr208es_767-780_dominquez.pdf

Meyn, A., White, P. S., Buhk, C., \& Jentsch, A. (2007). Environmental drivers of large, infrequent wildfires: The emerging conceptual model. Progress in Physical Geography, 31(3), 287-312. doi:10.1177/0309133307079365

Mondal, N., \& Sukumar, R. (2016). Fires in seasonally dry tropical forest: Testing the varying constraints hypothesis across a regional rainfall gradient. PLOSONE, 11(7), 1-16. doi:10.1371/journal.pone.0159691

Myers, R.L. (2006). Convivir con el fuego -Manteniendo los ecosistemas y los medios de subsistencia mediante el manejo de integral del fuego. Iniciativa global para el manejo del fuego. Florida: The Nature Conservancy.

Otterstrom, S., Schwartz, M., \& Velázquez-Rocha, I. (2006). Responses to Fire in Selected Tropical Dry Forest Trees. Biotropica, 38(5), 592-598. Recuperado de: http://onlinelibrary.wiley.com/ doi/10.1111/j.1744-7429.2006.00188.x/full

Portillo-Quintero, C. A., \& Sanchez-Azofeifa, G. A. (2010). Extent and conservation of tropical dry forests in the Americas. Biological Conservation, 143(1), 144-155. doi:10.1016/j. biocon.2009.09.020

Sánchez-Azofeifa, A., Calvo-Alvarado, J., Marcos do EspíritoSanto, M., Fernandes, G., \& Powers, J. (2013). Tropical Dry Forests in the Americas. Tropical Dry Forests in the Americas, 1-15. doi:10.1201/b15417-2

Sánchez-Azofeifa, G.A., Guzmán-Quesada, J. A., Vega-Araya, M., Campos-Vargas, C., Durán, S.M., D'Souza, W., Gianoli, T., Portillo-Quintero, C., \& Sharp, I. (2017). Can terrestrial laser scanners (TLSs) and hemispherical photographs predict tropical dry forest succession with liana abundance?. Biogeosciences, 14, 977-988. doi: 10.5194/ bg-14-977-2017

Schmerbeck, J. (2011). Linking dynamics and locally important ecosystem services of South Indian dry forests: an approach. Journal of Resources, Energy and Development, 8(2), 149-172. Recuperado de http://www.indianjournals.com/ijor. aspx?target=ijor:read\&volume $=8 \&$ issue $=2 \&$ article $=007$

Ulate Quesada, C. (2011). Análisis y comparación de la biomasa aérea de la cobertura forestal según zona de vida y tipo de bosque para Costa Rica. (Tesis de licenciatura). Instituto Tecnológico de Costa Rica. Cartago, Costa Rica.

Van Bloem, S., Murphy, P., \& Lugo, A. (2004). Tropical dry forests. Encyclopedia of Forest Sciences, 24(1), 1767-1775. doi:10.1016/B0-12-145160-7/00176-9

Vargas-Sanabria, D. (2016). Dinámica del paisaje en áreas afectadas por incendios forestales en el bosque tropical seco del Área de Conservación Guanacaste, Costa Rica. (Tesis de licenciatura). Universidad Estatal a Distancia. San José, Costa Rica.

Vargas-Sanabria, D., \& Campos- Vargas, C. (2018). Sistema multi-algoritmo para la clasificación de coberturas de la tierra en el bosque seco tropical del Área de Conservación Guanacaste. Tecnología en Marcha. 31 (1), 58-69. doi:10.18845/tm. v31i1.3497 